PAPER

\title{
Validation of a short disease specific quality of life scale for hemifacial spasm: correlation with SF-36
}

\author{
E K Tan, S Fook-Chong, S-Y Lum, J Thumboo
}

J Neurol Neurosurg Psychiatry 2005;76:1707-1710. doi: 10.1136/jnnp.2005.065656

See end of article for authors' affiliations

......................

Correspondence to: Dr Eng-King Tan, Department of Neurology, Singapore General Hospital, Outram Road Singapore 169608 gnrtek@sgh.com.sg

Received 15February 2005 Revised version received 20 April 2005 Accepted 26 April 2005
Background: A short, practical, and validated quality of life (QoL) scale for hemifacial spasm (HFS) is not currently available.

Objectives: To examine the reliability and validity of a short self-rating scale (HFS-7) by comparing HFS patients with healthy controls. We also evaluated the correlation of HFS-7 with the physical and mental domains of SF-36, a generic QoL scale.

Methods: Seven self-rating items (HFS-7) were administered to HFS patients and healthy controls. In addition, HFS patients answered the SF-36 questionnaire. The validity and reliability of HFS-7 were analysed and correlation between HFS-7 and SF-36 examined.

Results: A total of 178 subjects were enrolled in the study, including $85 \mathrm{HFS}$ patients with mean age of 54.8 (SD 11.0) years, of whom 52 (61.2\%) were women, and 93 controls with mean age of 51.4 (SD 10.0) years, of whom 59 (63.4\%) were women. The test-retest intraclass correlation coefficient for the seven items was between 0.75 and 0.90 and Cronbach's coefficient of reliability for the HFS-7 scale was 0.88. Every item in HFS-7 discriminated between disease and controls $(p<0.0001)$. The HFS-7 summary index correlated with the SF-36 summary score (Spearman's correlation $r=-0.28, p=0.009$ ), in particular the mental health summary score $(r=-0.416, p<0.0001)$ and the emotional domain $(\mathrm{r}=-0.466, \mathrm{p}<0.00001)$.

Conclusion: HFS-7 could prove useful as a simple clinical tool to assess and monitor QoL measures in HFS patients.
$\mathrm{H}$ emifacial spasm (HFS) is the involuntary contractions of the facial muscles innervated by the ipsilateral facial nerve, ${ }^{12}$ and is commonly due to an underlying neurovascular compression of the root exit zone of the facial nerve. $^{34}$ Botulinum toxin provides an effective medical treatment. ${ }^{5}$

Since HFS is a chronic condition that affects the facial muscles, it frequently leads to social embarrassment. In severe cases, the symptoms can affect vision, speech, and mental concentration. Such problems invariably impact on the patient's perception and satisfaction with various aspects of their life. ${ }^{67}$ Quality of life (QoL) is an important outcome measure in chronic diseases since it is a predictor of morbidity and mortality. ${ }^{8}$

QoL evaluation for HFS has been unsatisfactory as validated disease specific scales that measure domains of QoL of importance to HFS patients are lacking. Since HFS patients frequently suffer from emotional and related mental problems rather than physical disability because of the nature of their illness, generic QoL scales may not capture the full impact of HFS on subjects' QoL. In a previous study, we demonstrated some good psychometric properties of a 30 item QoL questionnaire for HFS patients. Some of the items examined were sensitive to response to botulinum toxin treatment. ${ }^{9}$ However, the questionnaire had a number of shortcomings: it was too lengthy, not all the items correlated with clinical severity or were sensitive to treatment response, and its discriminant validity between patients and controls and its correlation with a generic QoL scale were not examined.

To address these issues, we examined the reliability and validity of a short self-rating scale (HFS-7) by comparing HFS patients and healthy controls. We also evaluated the correlation of HFS-7 with the physical and mental domains of SF-36, a generic QoL scale. ${ }^{10}$

\section{METHODS}

A total of 178 subjects including HFS patients and controls were recruited for this cross sectional validation study.

\section{Patient selection}

Eighty five consecutive patients in a tertiary referral centre diagnosed as having HFS by a movement disorder specialist using standardised clinical criteria $^{12}$ were included. The following were the inclusion criteria: (a) a clinical diagnosis of primary HFS; and (b) no evidence of cognitive dysfunction based on a Mini-Mental State Examination. Those patients with chronic debilitating or life threatening diseases (such as organ failure or metastatic malignancies) or other movement disorders (such as blepharospasm or parkinsonism) were excluded. Seventy of the patients had previously participated in our earlier evaluation of the 30 item questionnaire.'

\section{Control selection}

Ninety three healthy controls (patients' spouses and healthy volunteers recruited from outpatient clinics) matched for age, gender, and race, and with no significant medical history consented to answer the HFS-7 anonymously. The controls were healthy volunteers with no medical illnesses requiring medication and no evidence of HFS on examination. These controls were informed that their responses would be compared to those of patients with involuntary facial spasms. The institutional ethics committee approved the work.

\section{HFS-7 questionnaire}

The selection of items for HFS-7 (table 1) was based on our previous study of a 30 item questionnaire. ${ }^{9}$ These seven items (in the English language) were from the domains mobility,

Abbreviations: HFS, hemifacial spasm; ICC, intraclass correlation coefficient; QoL, quality of life; SI, summary index 


\begin{tabular}{|lll|}
\hline $\begin{array}{l}\text { Table } 1 \\
\text { controls }\end{array}$ & Characteristics of HFS patients and healthy \\
\hline & HFS patients & Healthy controls \\
\hline Number & 85 & 93 \\
Mean (SD) age, years & $54.8(11.1)$ & $51.4(10.0)$ \\
Female, $n$ & $52(61.2 \%)$ & $59(63.4 \%)$ \\
Severity of HFS, grade & & \\
1 & $2(2.4 \%)$ & \\
2 & $15(17.6 \%)$ & \\
3 & $54(63.5 \%)$ & \\
4 & $14(16.5 \%)$ & \\
& & \\
\end{tabular}

activities of daily living, emotional well-being, and stigma. Six of the seven items were selected from 10 items which, based on our statistical analysis in a previous study, have been shown to be most sensitive to response to botulinum toxin injection in our HFS patients. ${ }^{9}$ These items, together with the seventh item "difficulty in reading", were also considered to be important by our patients, based on our previous clinical interviews. ${ }^{9}$

All the items were scored on a five point scale ranging from 0 ("never") to 4 ("always"). Patients were asked to answer the self-rating questions on two separate occasions (23 week interval) in the outpatient clinic. The answers to the questions were based on how the patients felt 2-3 weeks prior to the date of the test. In addition, all patients also answered the SF-36 questionnaire, a generic QoL scale. ${ }^{9}$

\section{SF-36 questionnaire}

This widely used validated questionnaire has 36 items. ${ }^{10}$ It covers eight domains broadly divided into physical health (physical functioning, physical role, bodily pain, and general health) and mental health (vitality, social functioning, emotional role, and mental health). We have validated the SF-36 in our population in a previous community based survey $(\mathrm{n}=5503) .{ }^{11}$

\section{Assessment of severity of HFS}

The severity of HFS in the patients was rated based on a five point scale (0: normal, 1: slight disability, 2: moderate disability, no functional impairment, 3: moderate disability, has functional impairment, and 4: severely incapacitated) by a movement disorder neurologist blinded to the scores on the HFS-7 and SF-36.

\section{Collection of data}

The physicians' assessment and the patients' self-rating scores were collected and entered into the computer database independently. Statistical analysis was carried out only after all data of the study subjects had been collected.

Table 2 Reliability of scale (test-retest): intraclass correlation coefficient

\begin{tabular}{ll}
\hline HFS-7 items & ICC $(95 \% \mathrm{CI})$ \\
\hline 1. Had difficulty driving & $0.77(0.49-0.9)$ \\
2. Had difficulty reading & $0.87(0.79-0.92)$ \\
3. Had difficulty watching television/movie & $0.86(0.77-0.92)$ \\
4. Felt depressed & $0.89(0.82-0.94)$ \\
5. Avoided eye contact & $0.84(0.73-0.9)$ \\
6. Felt embarrassed about having the condition & $0.90(0.82-0.94)$ \\
7. Felt worried about other's reactions to you & $0.75(0.58-0.85)$ \\
\hline
\end{tabular}

\section{Statistical analysis}

Internal consistency of each subscale was measured using Cronbach's $\alpha$ coefficient. We assessed the test-retest reliability of each item of the HFS-7 by calculating the intraclass correlation coefficient (ICC). Cronbach's $\alpha$ and ICC ${ }^{12}$ greater than 0.7 would be regarded as indicating good reliability. Landis and $\mathrm{Koch}^{12}$ derived a kappa-type statistic $(\kappa)$ and classified the strength of agreement as substantial and perfect for $\kappa$ in the ranges (0.61-0.80) and (0.81-1.00), respectively. As ICC and $\kappa$ are analogous, we decided on the cut off of 0.7. Correlation of severity of HFS with the questionnaire was measured using Spearman's rank correlation coefficient. The rating scores for the items being on a five point scale were ordinal and thus non-parametric tests were deemed more suitable. All the correlations reported as significant were linear and each Spearman's correlation coefficient was very close to the corresponding Pearson correlation coefficient.

Median and interquartile range (25th and 75 th percentile) summary statistics for the item scores of HFS-7 were presented for HFS patients and controls, and the scores for the two groups were compared using the Mann-Whitney test.

A summary index (SI) ranging from 0 to 100 was created for the seven questions of the HFS-7. This SI was calculated as follows: HFS-7 SI $=$ sum of scores of the seven questions $\times 100 /$ maximum score per question multiplied by 7 . The mean of the physical health and mental health domain scores of SF-36 was also calculated. We referred to this mean score with a range of $0-100$ as the SF-36 physical or mental SI. The physical health domains of SF-36 were physical functioning, physical role, bodily pain, and general health. The mental health domains were vitality, social functioning, emotional role, and mental health. We analysed the following correlations: HFS-7 SI with the overall SF-36 SI; HFS-7 SI with SF36 physical health SI and SF-36 emotional health SI; mental health items (items 4-6) of HFS-7 with SF-36 mental health domains; and physical health items of HFS-7 (items 1-3) with the physical health domains of SF-36. Sixteen comparisons were investigated for the purpose of correlating mental items of HFS-7 with mental domains of SF-36. Thus we adjusted for multiple comparisons by testing each correlation at $p<0.003$. SPSS for Windows version 10.1 was used for data analysis.

\section{RESULTS}

The 178 subjects comprised 85 HFS patients with a mean age of 54.8 (SD 11.0) years and including 52 (61.2\%) women, and 93 controls with mean age of 51.4 (SD 10.0) years and including $59(63.4 \%)$ women (table 1$)$. The majority of HFS patients had left-sided symptoms, with a mean duration of symptoms of 3.8 (SD 3.0) years (range 0.5-15). The mean stage of severity of HFS was 2.5 (SD 0.6) (range 1-4).

The response rate for the questionnaire survey was $100 \%$. The ICC for each of the seven items was between 0.75 and 0.90 and Cronbach's $\alpha$ was 0.88 (table 2). Each of the seven items in HFS-7 discriminated between disease and controls (table 3). The proportion of HFS patients who reached the ceiling and floor effect for the seven items is given in table 3. There was a positive correlation of severity with HFS-7 SI $(\mathrm{r}=0.319, \mathrm{p}=0.003)$.

A good functional outcome is indicated by a high score for the SF-36 but by a low score for the HFS-7. We showed that the HFS-7 SI correlated with the SF-36 summary score (mean of the physical health and mental health score) $(\mathrm{r}=-0.28$, $\mathrm{p}=0.009$ ). Subset analysis demonstrated that there was robust association of the HFS-7 SI with the SF-36 mean mental health SI $(\mathrm{r}=-0.416, \mathrm{p}<0.0001)$. This was particularly true for the domains of social functioning $(r=-0.260$, $p=0.017)$, emotional role $(r=-0.466, p<0.0001)$, and 
Table 3 Comparison of HFS-7 item scores between HFS patients and controls

\begin{tabular}{|c|c|c|c|c|c|c|}
\hline Item & Disease group & Median & $\mathrm{IQR}^{*}$ & $p$ value & $\%$ reaching floor & $\%$ reaching ceiling \\
\hline \multirow[t]{2}{*}{ 1. Had difficulty driving } & Control & 0 & 0,0 & $<0.00001$ & - & - \\
\hline & HFS & 0 & $0,1.5$ & & 4.7 & 58.1 \\
\hline \multirow[t]{2}{*}{ 2. Had difficulty reading } & Control & 0 & 0,0 & $<0.00001$ & - & - \\
\hline & HFS & 2 & 1,3 & & 11.9 & 14.3 \\
\hline \multirow[t]{2}{*}{ 3. Had difficulty watching television/movie } & Control & 0 & 0,0 & $<0.00001$ & - & - \\
\hline & HFS & 2 & 1,3 & & 8.3 & 21.4 \\
\hline \multirow[t]{2}{*}{ 4. Felt depressed } & Control & 0 & 0,0 & $<0.00001$ & - & - \\
\hline & HFS & 1 & 0,2 & & 4.8 & 39.3 \\
\hline \multirow{2}{*}{ 5. Avoided eye contact } & Control & 0 & 0,0 & $<0.00001$ & - & - \\
\hline & HFS & 2 & $1.5,4$ & & 26.2 & 13.1 \\
\hline \multirow[t]{2}{*}{ 6. Felt embarrassed about having the condition } & Control & 0 & 0,0 & $<0.00001$ & - & - \\
\hline & HFS & 2 & $1.5,4$ & & 26.5 & 12.0 \\
\hline \multirow[t]{2}{*}{ 7. Felt worried about other's reactions to you } & Control & 0 & 0,0 & $<0.00001$ & - & - \\
\hline & HFS & 2 & $1.5,3$ & & 23.8 & 14.3 \\
\hline
\end{tabular}

mental health $(\mathrm{r}=-0.264, \mathrm{p}=0.015)$, but not for the physical health SI $(\mathrm{r}=-0.17, \mathrm{p}=0.13)$. The mental health items 4, 5, 6, and 7 of HFS-7 were significantly associated with the mental health domains of SF-36 (table 4). However, the physical health items 1, 2, and 3 of HFS-7 did not correlate with the physical domains of SF-36. The distribution of scores for each item of HFS-7 and HFS-7 SI by severity of HFS is summarised in table 5. The scores for the items and SI increased with worsening severity of HFS. Linear trends were observed for all the significant correlations in the study.

\section{DISCUSSION}

Validated health related QoL scales frequently employed for other neurological diseases can not be reliably applied to HFS. This is because the physical problems of HFS are frequently limited to visual impairment, and emotional distress and fear of social stigmatisation are the primary causes of patients' perception of poor QoL. ${ }^{9}$ Generic QoL scales generally give limited importance to these specific features of HFS. ${ }^{6}$ Therefore, if generic questionnaires are used, HFS patients could be answering irrelevant questions that may not be sensitive to changes in outcome. Ideally, an HFS specific QoL scale should be short and concise and possess psychometric properties of validity, reliability, and high sensitivity for detecting changes. ${ }^{67}$ The reliability of the questionnaire is measured by examining internal consistency and test-retest variability.

In our earlier study, we developed a broad and encompassing range of 30 self-rating questions based on in-depth interviews with HFS patients and their family members who highlighted their particular health concerns." While we demonstrated that the overall questionnaire had some good psychometric properties, we found that a number of the items had high ceiling values and were not sensitive enough to clinical response. ${ }^{9}$ Furthermore, most patients found the questionnaire too lengthy. Hence we selected from the original questionnaire seven items (HFS-7) which covered the important domains as perceived by our patients, ${ }^{9}$ were at the same time significantly correlated with the severity of HFS, and were sensitive to a change in clinical response to

Table 4 Distribution of item scores and SI of HFS-7 by severity

\begin{tabular}{|c|c|c|c|c|c|c|c|}
\hline HFS-7 item/SI & Severity* & $\mathbf{n}$ & Median & Min & Max & $\begin{array}{l}25 \text { th } \\
\text { percentile }\end{array}$ & $\begin{array}{l}\text { 75th } \\
\text { percentile }\end{array}$ \\
\hline \multirow[t]{3}{*}{ HFS-7 SI } & 2 & 15 & 28.6 & 10.7 & 71.4 & 21.4 & 42.9 \\
\hline & 3 & 54 & 42.9 & 0.0 & 100.0 & 21.4 & 60.7 \\
\hline & 4 & 14 & 64.3 & 10.7 & 85.7 & 43.8 & 75.9 \\
\hline \multirow[t]{3}{*}{ 1. Had difficulty driving } & 2 & 4 & 0 & 0 & 0 & 0 & 0 \\
\hline & 3 & 29 & 0 & 0 & 4.0 & 0 & 2.0 \\
\hline & 4 & 8 & 0 & 0 & 4.0 & 0 & 2.5 \\
\hline \multirow[t]{3}{*}{ 2. Had difficulty reading } & 2 & 15 & 2.0 & 0 & 4.0 & 1.0 & 2.0 \\
\hline & 3 & 54 & 2.0 & 0 & 4.0 & 1.0 & 2.5 \\
\hline & 4 & 14 & 3.0 & 0 & 4.0 & 2.0 & 4.0 \\
\hline \multirow{3}{*}{ 3. Had difficulty watching television/movie } & 2 & 15 & 1.0 & 0 & 3.0 & 0 & 2.0 \\
\hline & 3 & 54 & 2.0 & 0 & 4.0 & 1.0 & 2.0 \\
\hline & 4 & 14 & 3.0 & 0 & 4.0 & 2.0 & 3.3 \\
\hline \multirow[t]{3}{*}{ 4. Felt depressed } & 2 & 15 & 1.0 & 0 & 4.0 & 0 & 2.0 \\
\hline & 3 & 54 & 2.0 & 0 & 4.0 & 0 & 2.0 \\
\hline & 4 & 14 & 0 & 0 & 4.0 & 0 & 3.0 \\
\hline \multirow[t]{3}{*}{ 5. Avoided eye contact } & 2 & 15 & 2.0 & 0 & 4.0 & 1.0 & 2.0 \\
\hline & 3 & 54 & 2.0 & 0 & 4.0 & 1.0 & 3.5 \\
\hline & 4 & 14 & 4.0 & 0 & 4.0 & 2.8 & 4.0 \\
\hline \multirow[t]{3}{*}{ 6. Felt embarrassed about having the condition } & 2 & 15 & 2.0 & 0 & 4.0 & 2.0 & 2.0 \\
\hline & 3 & 54 & 2.0 & 0 & 4.0 & 1.0 & 3.0 \\
\hline & 4 & 14 & 4.0 & 0 & 4.0 & 2.8 & 4.0 \\
\hline \multirow[t]{3}{*}{ 7. Felt worried about other's reactions to you } & 2 & 15 & 2.0 & 0 & 4.0 & 2.0 & 2.0 \\
\hline & 3 & 54 & 2.0 & 0 & 4.0 & 1.0 & 3.0 \\
\hline & 4 & 14 & 3.5 & 0 & 4.0 & 2.0 & 4.0 \\
\hline
\end{tabular}

*Severity grading: 0, normal; 1, slight disability; 2, moderate disability, no functional impairment; 3, moderate disability, functional impairment; 4, severely incapacitated.

Only two HFS patients had slight disability and none were normal. Data for these two grades of severity are not displayed in the table. The sample size was less for item 1 because non-drivers and those who did not drive during the 2-3 weeks prior to answering the questionnaire, did not respond to the question. 
Table 5 Correlation of mental health items of HFS-7 with SF-36 mental health domains

\begin{tabular}{lllll}
\hline Item & Vitality & Social functioning & Emotional role \\
\hline Felt depressed & -0.14 & $-0.33^{*}$ & -0.26 & -0.22 \\
Avoided eye contact & -0.21 & -0.27 & $-0.44^{*}$ & -0.23 \\
Felt embarrassed about having the condition & -0.22 & -0.24 & $-0.47^{*}$ & $-0.45^{*}$ \\
Felt worried about other's reactions to you & -0.11 & -0.24 & -0.22 & \\
\hline *Significant at $\mathrm{p}<0.003$. & & &
\end{tabular}

botulinum toxin treatment. To address content validity, HFS7 comprised predominantly of items related to social stigma and emotional problems. In addition, items in domains of activities of daily living and mobility, such as reading, watching television, and driving, were included.

Using a case control methodology in this present study, we demonstrated that HFS-7 has discriminating power to differentiate HFS patients from healthy subjects since there were highly significant differences in the response to all seven items between HFS patients and controls. In addition, there was a good correlation of the HFS-7 SI with the overall SF-36 SI, and in particular the SF-36 mental health SI. Specifically, there was correlation of HFS-7 SI with the emotional and, to a lesser extend, the social functioning and mental health domains. The four mental health items of HFS-7 also showed correlation with the SF-36 mental health domains. However, there was a lack of association of the physical items of HFS-7 with the physical health domains of SF-36. This was not surprising as physical problems specific to HFS patients, such as having difficulty in reading and watching television, were not measured in SF-36. Hence development of a disease specific QoL scale for HFS is of clinical importance.

We also demonstrated that a low proportion of patients (12-21\%) for five of the seven items reached the ceiling value. Lastly, we replicated our earlier finding ${ }^{9}$ that these items have good internal consistency (Cronbach's $\alpha>0.75$ ) and test-retest reliability (ICC $>0.75$ ). There was a significant positive correlation of most of the subscale scores with the severity of HFS.

Our study has some potential limitations. While we demonstrated validity of HFS-7, cautious interpretation regarding the correlation with SF-36 is advised. In the absence of a real gold standard measure, perhaps comparison with SF-36 is not strictly necessary. However, the moderate correlation coefficients we demonstrated suggest that there is at least some degree of association of our QoL scale with SF36 , a widely used and validated general health related QoL instrument. The scores in HFS-7 were not intended to be used as a diagnostic tool and hence the absolute scores may have limited clinical utility.

In conclusion, this is the first demonstration (to our knowledge) of good discriminant validity of a short and simple QoL scale (HFS-7) covering the important problems specific to HFS. There was correlation of HFS-7 with the mental health component of the generic SF-36 scale. We have also demonstrated reliability of the items in HFS-7. Hence we suggest that the scale could prove useful as a simple clinical tool to assess and monitor QoL measures in HFS patients.

\section{ACKNOWLEDGEMENTS}

The authors thank the Department of Neurology, Singapore General Hospital for support.

\section{Authors' affiliations}

E K Tan, S-Y Lum, Department of Neurology, Singapore General Hospital, Singapore

S Fook-Chong, Department of Clinical Research, Singapore General Hospital, Singapore

J Thumboo, Department of Rheumatology, Singapore General Hospital, Singapore

Competing interests: none declared

\section{REFERENCES}

1 Wang A, Jankovic J. Hemifacial spasm: clinical findings and treatment. Muscle Nerve 1998;21:1-8.

2 Tan NC, Chan LL, Tan EK. Hemifacial spasm and involuntary facial movements. QJM 2002;95:493-500.

3 Janetta PJ. Cranial rhizopathies. In: Neurological surgery. 3rd ed. Philadelphia, PA: WB Saunders, 1990:4169-82.

4 Tan EK, Lum SY, Koh P, et al. Is hemifacial spasm associated with hypertension? Neurology 2003;60:343-4.

5 Jankovic J, Brin MF. Therapeutic uses of botulinum toxin. N Engl J Med 1991;324:1186-94.

6 Meyers AR, Gage H, Hendricks A. Health-related quality of life in neurology. Arch Neurol 2000:57:1224-7.

7 Marinus J, Ramaker C, van Hilten JJ, et al. Health related quality of life in Parkinson's disease: a systematic review of disease specific instruments. J Neurol Neurosurg Psychiatry 2002;72:241-8.

8 Idler EL, Benyamini Y. Self-rated health and mortality: a review of twentyseven community studies. J Health Soc Behav 1997;38:21-37.

9 Tan EK, Fook-Chong S, Lum SY, et al. Botulinum toxin improves quality of life in hemifacial spasm: validation of a questionnaire (HFS-30). J Neurol Sci 2004;219(1-2):151-5.

10 Ware JE Jr, Snow KK, Kosinski M, et al. SF-36 Health Survey manual and interpretation guide. Boston, MA: Nimrod, 1993.

11 Thumboo J, Fong KY, Machin D, et al. A community-based study of scaling assumptions and construct validity of the English (UK) and Chinese (HK) SF-36 in Singapore. Qual Life Res 2001;10(2):175-88.

12 Landis JR, Koch GG. The measurement of observer agreement for categorical data. Biometrics 1977;33:159-74. 